\title{
Analysis of Chinese College Students' Attitudes towards Brain Computer Interface
}

\author{
Ma Lan \\ Marxism School, Jianghan University, Wuhan 430056, China \\ lanhorse@163.com
}

Keywords: brain computer interface; ethics of BCI; informed consent; privacy

\begin{abstract}
Brain Computer Interface (BCI) is a rapidly developing area of neuroscience research, which makes direct communication between the brain and a machine possible by means of electrodes. This paper presents relevant ethical problems that are likely to emerge in the following decades. Over the discussion of BCI, the views of college students have been largely neglected from the discussion. It is necessary to analyze the attitudes of college students in China. The data collected suggest that college students nowadays in China are interested in the BCI and tend to cautiously accept BCI with the purpose of social development even as they recognize its potential risk. These data might be helpful to various ethical arguments to the BCI, and interdisciplinary discussion, especially if seeking to the public view in China.
\end{abstract}

\section{Introduction}

Although we now face global issues, much of our activities are concerned with the emerging technology, such as information technology, neuroscience, and psychology. Regarding BrainComputer Interface, neuroscience provides a considerable amount of data applied in medicine and education. The development and deployment of Brain Computer Interface technology have proposed some ethical disputes and social issues in professional and academic field, such as informed consent, responsibility, privacy, safety and risk-benefit, justice. Individual assessments of the ethical implications of research and applications in a multi-disciplinary and multi-faceted area.

BCI researchers may have good reason for their reluctance to wade into ethics. The questions raised are likely to be open-ended, and their research both inside and outside the laboratory may be supported by some projects. Furthermore, many BCI scientists come from an engineering background, and may have less training and interest in ethical matters. However, BCI researchers may be interested in ethical issues they are confronted with in daily work situations, while some ethicists focus on more abstract, but no less relevant, themes like enacted mind or embodied mind. Paul and Gaye emphasize the complexity of ethical considerations of BCI and the need for further discussion. They all highlight some of the ethical, legal and social issues identified by stakeholders. [1] Nijboer and Clausen assessed respondents' opinions about BCI. [2] Over the discussion of BCI, the views of university students that have been largely neglected from the discussion. It is necessary to analyze the attitudes of the university students in China. To investigate the opinions about ethical 
issues related to BCI research for the development of assistive technology, we asked respondents to analysis the university students' attitudes towards the BCI.

\section{Experimental Methods}

To ensure the effective data for the samples, we have interviewed 10 university students including 24 male college students and 25 female college students with ages from 19 to 22 years living in different universities in different cities in China, and from different major background of history, literature, computer, and electronic information and different family. Of course, were presented with a brief demographic questionnaire though e-mail and they tend to accept this kind of questionnaire survey. Participants are provided informed consent and we used a semi-structure design, with a list of open questions. Additionally, we give different optional context for participants to explain the reason behind their choices. To confirm the participants fully understand the context of the survey, we have a communication through telephone to ensure them understand capacities. Data were analyzed using SPSS. The questions what we design in the survey and the results are as follows:

(1) Do you know what is BCI?

Yes or no.

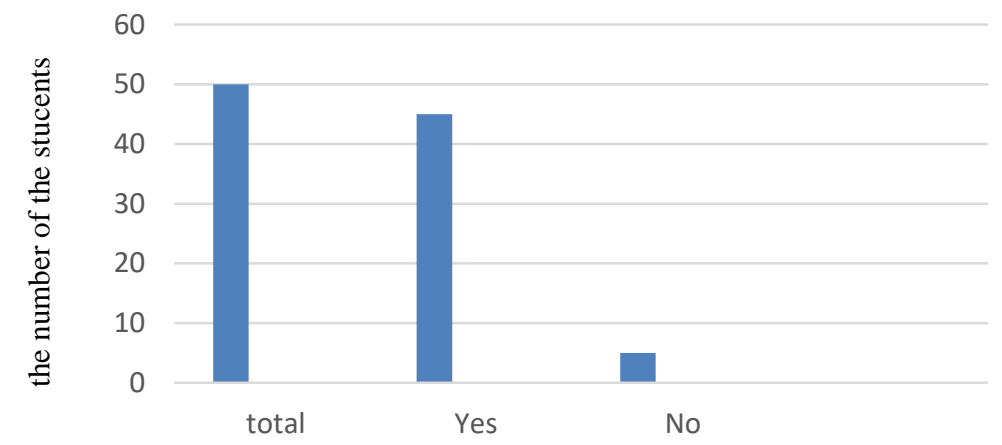

Fig.1 Do you know what is BCI?

(2) Do you pay attention to the ethical issues of BCI?

Yes or no.

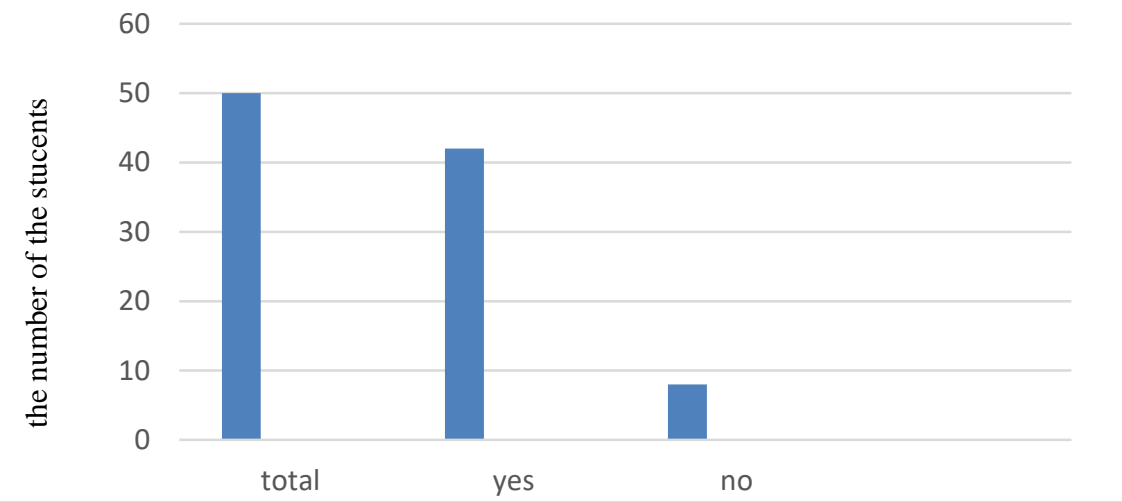

Fig. 2 Do you pay attention to the ethical issues of BCI?

(3) What kind of ethical issues of BCI you pay attention to?

Informed consent, responsibility, privacy, user safety, risk-benefit, justice, the human identity. 


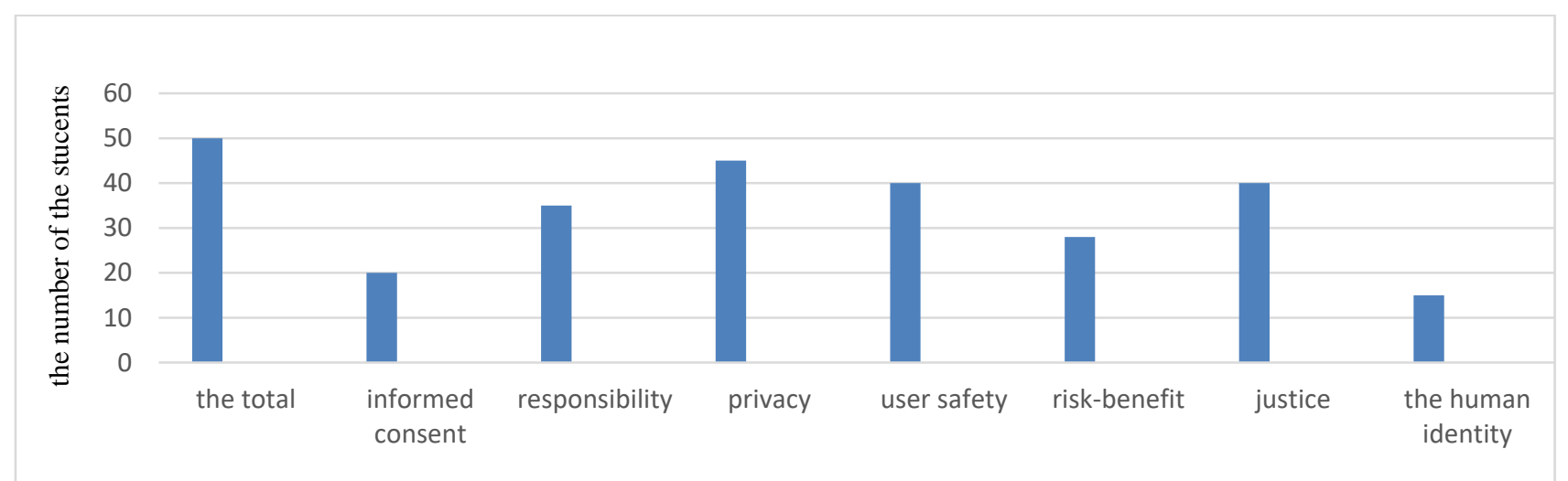

Fig. 3 What kind of ethical issues of BCI you pay attention to?

(4) Are you optimistic or pessimistic to the BCI?

Optimistic or pessimistic

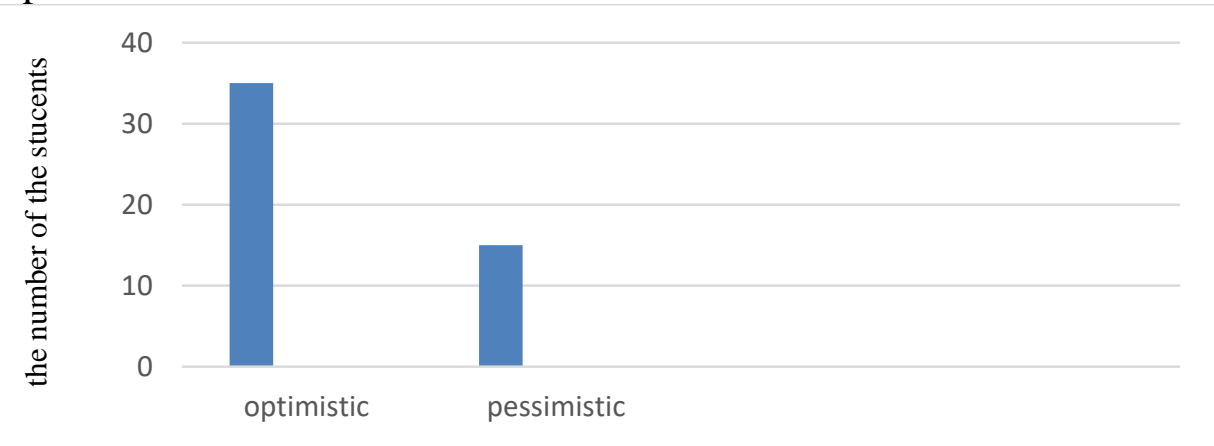

Fig. 4 Are you optimistic or pessimistic to the BCI?

\section{Analysis and Results}

\subsection{Concept of BCI}

The first graph shows that 45 university students of $90 \%$ participants understand BCI. BCI is a device that translates signals from the brain into a useful outcome without the use of muscles. Referring to the application of BCI, they know that BCI is a technology used for people with Parkinson's disease, essential tremor, and dystonia. Most of the university students understand that BCI is extremely complex medical device systems, requiring expertise and input from neuroscientists, engineers, biochemists, medical practitioners, and others. Most of them pay attention to the media report about BCI and a few of them will spend time on the books of BCI. Though 5 university students of 10\% participants don't know the exact concept of BCI, it doesn't mean they haven't heard of BCI as an emerging technology.

\subsection{Attitudes When Referring Participants Themselves}

The second graph showed that 42 university students of $84 \%$ participants are concerned with the ethical issues of BCI. On the contrary, 8 university students of 16\% participants don't understand and pay attention to the ethical challenges raised by the BCI. Let us go to analyses the third and the fourth graph. We might find what kinds of ethical issues they focus on most, and what their attitudes to the development of BCI are though there are many ethical issues. 


\subsection{Informed Consent, Responsibility, Privacy, User Safety, Risk-Benefit, Justice, Human Identity}

Those who are for the BCI argue that the benefit the individuals gained from the BCI expand all kinds of chance in medicine and social competition. However, it is showed that 20 college students of the total 50 care about informed consent. BCI is an experimental treatment and its therapeutic viability has partly been proven. If the patients are informed about all the process of treatment and consent the treatment, BCI is an effective method for their pursuit of the better life. Of course, It is the researchers' responsibility to ensure the informed consent during process of the treat to disease and enhance capability. From the pragmatics standpoint, healthy body and extended human's ability are viewed as indispensable in the course of recognition and exploration of the world. 70\% university students of the participants pay attention to the responsibility of the researchers and government. Most of the respondents are confident that BCI researchers are responsible for the executed actions and transmitted messages created with the aid of a BCI device.

Those who are against the BCI have several reasons provided. Among them the privacy and user safety of BCI the primary fears. Referring to privacy,45 university students of the 50 think there is a potential for new violations of user privacy. BCI devices could reveal a variety of information, ranging from truthfulness, to psychological traits and mental states, to attitudes toward other people. Protection of privacy is deemed extremely important when considering implementation of BCI technology. The proportion is higher than other reasons. The participants also worry about the direct risk of harm to the user, especially for devices that require surgical interventions. Though the participants are not the experts of the BCI, they consider the balance between benefit and risk. They suggest BCIs is an alternative assistive technology or the determination of their appropriateness for a given patient. General scientific uncertainty plays a role, but scientists haven't provided sufficient data or studies on the relative risk-benefit analysis of BCI. Therefore, the idea of an acceptable expectation of benefit may currently be unrealistic. They do not hesitate to describe BCI as an inherently risky technology. Referring to the justice, they also show the competition between the students who can afford the BCI as a kind of cognitive enhancement and it can improve their memory and those who can't afford it. Privileged students already enjoy many advantages over their less wealth peer. In China, inequality is a general social phenomenon in education. When analyzing the attitudes towards justice, 40 students of the 50 are concerned with justice of BCI, and only 28 participants are concerned with risk-benefit. Morever,30\% of the participants think human identity is emergent ethical issue. However, more western college students think human identity is important. The dignity of living a real life embodies the human identity. Some material operated in your brain change your own personal identity. Those who use the BCI are unnatural and lack of human identity.

\section{Summary}

From the investigation and analyses, it is obvious that university students pay attention to the ethical issues of BCI. And they provide their views against or for the BCI. Though they worry about all kinds of challenges raised by BCI,35 students of 50 are optimistic to the development of BCI, which is higher than the pessimistic people.

\section{References}

[1] Paul McCullagh, Gaye Lightbody, Ethical Challenges Associated with the Development and Deployment of Brain Computer Interface Technology, Ethical Challenges Associated with BCI Development, Neuroethics . 7(2014):109-122. [2] Femke Nijboer,Jen Clausen, The Asilomar Survey: Stakeholders' Opinions on Ethical Issues Related to BrainComputer Interfacing, Neuroethics. 6(2013):541-578. 